\title{
Dispersion Modelling of Traffic Related Pollutants from Highways
}

\author{
Lamiaa Khalid and Shirook Ali \\ Sheridan College \\ Brampton, Ontario, Canada \\ shirook.ali@sheridancollege.ca
}

\begin{abstract}
In this paper, we use the CAL3QHC air dispersion model to predict the concentration of air pollutants of interest at desired locations. We utilize the U.S. Environmental Protection Agency (EPA) Motor Vehicle Emission Simulator (MOVES) to generate the emission levels used in our analysis. Using the output from the air dispersion model, we will develop site-specific emission information. This information will be used to map out the vertical and horizontal placement of the sensors for optimal sensor deployment. The deployed sensors will be used to monitor the concentrations of traffic related pollutants near highways in real time.
\end{abstract}

Keywords: Dispersion Modelling, CAL3QHC, EPA MOVES, Air Quality Monitoring.

\section{Introduction}

Transportation is one of the largest sources of air pollutants in Canada. In Ontario, traffic sources contribute significantly to the total amount of pollutants in the atmosphere (up to $80 \%$ in the case of carbon monoxide). Emissions from motor vehicle operations near major roads have led to elevated concentrations of certain air pollutants, including carbon monoxide $(\mathrm{CO})$; nitrogen oxides $\left(\mathrm{NO}_{\mathrm{X}}\right)$; nitrogen dioxide $\left(\mathrm{NO}_{2}\right)$; particular matter $(\mathrm{PM})$; black carbon $(\mathrm{BC})$; and benzene near large roadways when compared with overall urban background levels [1,2]. It was shown in [3] that the overall concentrations of known mobile source emitted gaseous $(\mathrm{CO})$ and particulate $(\mathrm{BC})$ pollutants were elevated near the road, and the concentrations of these pollutants generally increased with increasing traffic activity.

There are currently two sets of air quality standards enforced in Canada: the National Ambient Air Quality Objectives (NAAQO) and Canadian Ambient Air Quality Standards (CAAQS). Both are benchmarks used to guide decisions on controlling air pollution emissions. The NAAQOs framework [3] specifies two levels developed through extensive scientific assessment: (i) a reference level, which is the level above which there are demonstrated effects on human health, and/or the environment; and (ii) an Air Quality Objective, which reflects a specific level of protection for the general population and environment and also considers aspects of technical feasibility. The CAAQS are stringent air quality standards for ground-level ozone and fine particulate matter because of their adverse effects on human health [4].

Since vehicular emissions are one of the major causes of high levels of air pollutants, it is important to assess traffic emissions and their impacts on air quality near heavily travelled highways. To evaluate the pollutant emissions of road transportation, vehicle emissions inventory needs to be calculated. It is very difficult to predict quantitatively vehicle emissions from first principles. Therefore, emission predictions are usually based on vehicle emission test results. The U.S. Environmental Protection Agency (EPA) annually tests a cross-section of vehicle models under controlled laboratory conditions. The test data generated on individual vehicles are used in the U.S. EPA emission simulation model Motor Vehicle Emission Simulator (MOVES) [5]. MOVES estimates emissions for on-road and non-road sources, covers a broad range of pollutants, and enables multiple-scale analysis from fine scale to national emission inventory scale.

Air dispersion models are used to describe the complex mixture of pollutants and show the effect of atmospheric transport mechanisms on pollutant dispersion near-highways. The largest emission factors for $\mathrm{CO}$ and other pollutants tend to occur at speeds of less than 20 miles per hour ( $\mathrm{mph}$ ) because of inefficient engine operation primarily due to stop/start activity and frequent idling/acceleration. $\mathrm{CO}_{2}$ emissions are linked directly to fuel consumption, so $\mathrm{CO}_{2}$ emissions per mile go up at very low or very high speeds. Knowledge of traffic-flow patterns is relevant because local pollutant concentrations are directly proportional to vehicle numbers and their characteristics. 
Numerous air quality models exist within routine air quality management and planning practice as well as within academic research for near-roadway applications as reviewed by the U.S. EPA which include computational fluid dynamic, Gaussian plume, geographic information system-based, puff, and statistical models [6].

CALINE3 [7] is a finite line source Gaussian dispersion model. CALINE-3 is designed to predict air pollutant concentrations near highways and arterial streets due to emissions from motor vehicles operating under free flow conditions. CAL3QHC, a variant of CALINE-3, incorporates methods for estimating queue lengths and the contribution of emissions from idling vehicles. The model permits the estimation of total air pollution concentrations from both moving and idling vehicles. Therefore, we will use the CAL3QHC model in our analysis as it is appropriate for estimating near-roadway micro-scale concentrations and has been previously validated.

CAL3QHC was developed primarily for CO hot-spot analyses, computing hourly concentrations using "worse case" meteorology, which can then be scaled to an 8-hour average to estimate compliance with the CO NAAQO. When dealing with inert pollutants, a Gaussian dispersion model will operate in the same way regardless of pollutant. While CAL3QHC is hard-coded to convert the input emissions to mixing ratios of $\mathrm{CO}$, the dispersion parameterizations in the model would apply for any pollutant. Therefore, the model's performance can be examined accurately using another inert pollutant. The required inputs for the CAL3QHC include roadway geometry, hourly surface meteorology, traffic volume and mission rates. Individual highway segments are divided into a series of elements, each modelled as an equivalent finite line source that is normal to the wind direction and centred at the element's midpoint, from which incremental concentrations are computed and summed to predict the concentrations at designated receptors. Pollutant concentration estimates are directly proportional to the emission factors used as input data to the program.

In our previous paper [8], we proposed a methodology that utilizes fixed and mobile sensor units to monitor pollution emission levels in real-time. We estimated the air pollution emission levels of highway traffic within an urban environment based on MOVES emission modelling system. We estimated the levels of the air pollutants of interest $\mathrm{CO}, \mathrm{NOx} \mathrm{CH}_{4}$ and hydrocarbons (HCs) for week days, and weekends at different hours of the day to reflect change in temperature and relative humidity, traffic volume and average speed. The goal of this paper is to utilize generated emission levels from MOVES to estimate the concentrations of the air pollutants of interest at desired locations using the CAL3QHC air dispersion model. Using the output from the air dispersion model, we will develop site-specific emission information. This information will be used to map out the sensor locations (vertical and horizontal placement) for optimal sensor deployment for monitoring the emission levels in real time.

The rest of the paper is organized as follows. We discuss the most relevant related work in section 2. We describe the integrated framework used to predict the pollutants concentrations in section 3 . We present the simulation results in section 4 and we conclude the paper in section 5

\section{Related Work}

Recently, an increasing amount of research focuses on integrating both traffic emission and dispersion analyses to investigate their chain reaction. The authors in [9] incorporated regional travel demand models and a microscopic traffic simulation model to estimate necessary traffic-related data. The output was streamed into a vehicle emission model to produce traffic emission data; then an air pollutant dispersion model was applied to calculate dispersion using both the traffic emission data and the estimated predominant wind direction that was obtained from a meteorological model. With the objective of analysing health impacts caused by heavy trucks, the authors in [10] combined a microscopic traffic simulation model with an air pollutant dispersion model to assess air quality and evaluate the impact of traffic emissions on public health.

Few studies have developed an integrated framework that handles both real-world traffic data and meteorological data to estimate traffic pollutant emissions and dispersion. The main challenges include data availability and modelling framework design. In [11], the authors proposed a novel air pollution estimation method that modelled traffic conditions, traffic-induced air pollution emissions and pollution dispersion. Their study focused on developing a prototype system. However, sensitivity analyses for modelling parameters were not thoroughly addressed in their study. The authors in [12] proposed a framework that incorporated conventional traffic and meteorological data to estimate traffic pollutant emission and dispersion over a large area using the MOVES model and the CALINE3 model. The MOVES model was used to generate the emission rates based on average vehicle speed on freeways and the CALINE3 air pollutant dispersion model was used to estimate pollutant dispersion along the regional freeway network using real world data. 


\section{Integrated Framework}

CAL3QHC permits the specification of up to 120 roadway links and 60 receptor locations within an XYZ plane. A new link is required when there is a change in link width, traffic volume, and travel speed or emission factor. The program automatically sums the contributions from each link to each receptor. In this work, we model a highway segment with a relatively stable average speed as a single link. We use a combination of vehicle fleet distribution (link source types: passenger cars, light and heavy duty trucks, motorcycles and buses) with fuel type formulation (gasoline or diesel). Based on the vehicle types, link volume and average speed, MOVES estimates the selected pollutants emission level produced on each link (grams/hour/link). Surface roughness and meteorological variables (such as atmospheric stability, wind speed and wind direction) are assumed to be spatially constant over the entire study area. Stability classes for six atmospheric stability classes are defined for CAL3QHC. Stability Class A refers to the most unstable condition while Stability Class F refers to the most stable atmospheric condition

The integrated framework is illustrated in Figure 1. This framework can be implemented to model real-time pollutant dispersion or predict future pollutant dispersion trends.

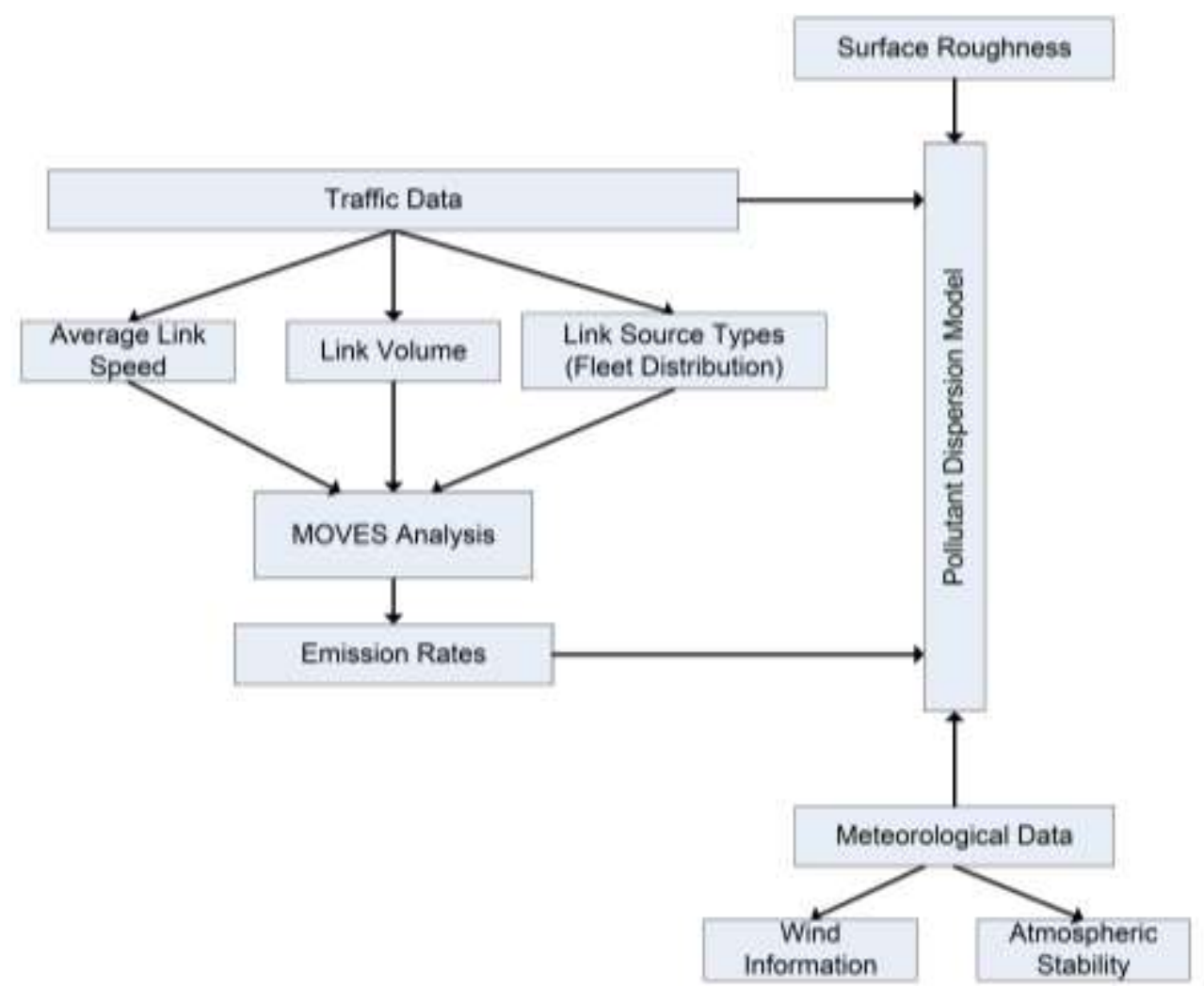

Fig. 1: Integrated Framework for MOVES Estimated Pollutant Emission and Dispersion Modelling.

\section{Simulation Results}

In this section, we first estimate the pollutant emission levels using MOVES2014a given the link average speed and traffic volume. We then couple those emission levels with atmospheric conditions (e.g., wind speed and direction and atmospheric stability) to estimate the pollutant concentration at various locations using the CAL3QHC dispersion model.

Data on seven-day hourly traffic volume and hourly average speed was obtained from Ministry of Transportation, Ontario (MTO) for two four-lane segments of the ON401, each of length $1.45 \mathrm{Km}$. Based on this data, the MOVES emission levels of different pollutants for the weekdays and weekends at different hours of the day for the month of April were estimated in [8].

Dispersion estimates based on CAL3QHC are computed on an hourly basis over the aggregate values of flow, wind speed, and wind direction per highway link. The link width is defined as the width of the travelled roadway (lanes of moving 
traffic only) plus 3 meters on each side to account for the dispersion of the plume generated by the wake of moving vehicles. The surface roughness is set to $100 \mathrm{~cm}$ (Suburban) and the atmospheric stability class is F (stable). Receptor (sensor) locations are specified in terms of X, Y, and Z coordinates. A receptor should be located outside the "mixing zone" of the free flow links (X coordinate) (i.e., total width of travel lanes plus 3 meters on each of the outside travel lanes. The receptors are spaced every $200 \mathrm{~m}$ along the length of the link (Y coordinate). The height of the receptors ( $\mathrm{Z}$ coordinate) is changed from $1.8 \mathrm{~m}$ (minimum height) to $50 \mathrm{~m}$ and the wind direction is changed from 225 degrees to 360 degrees (north wind) at different wind speeds to account for the impact of meteorology on pollutant concentrations. Background pollutant concentration was not considered since the purpose of this study is to assess traffic-related emissions. Background concentrations will be reflected in monitor readings later on in the project.

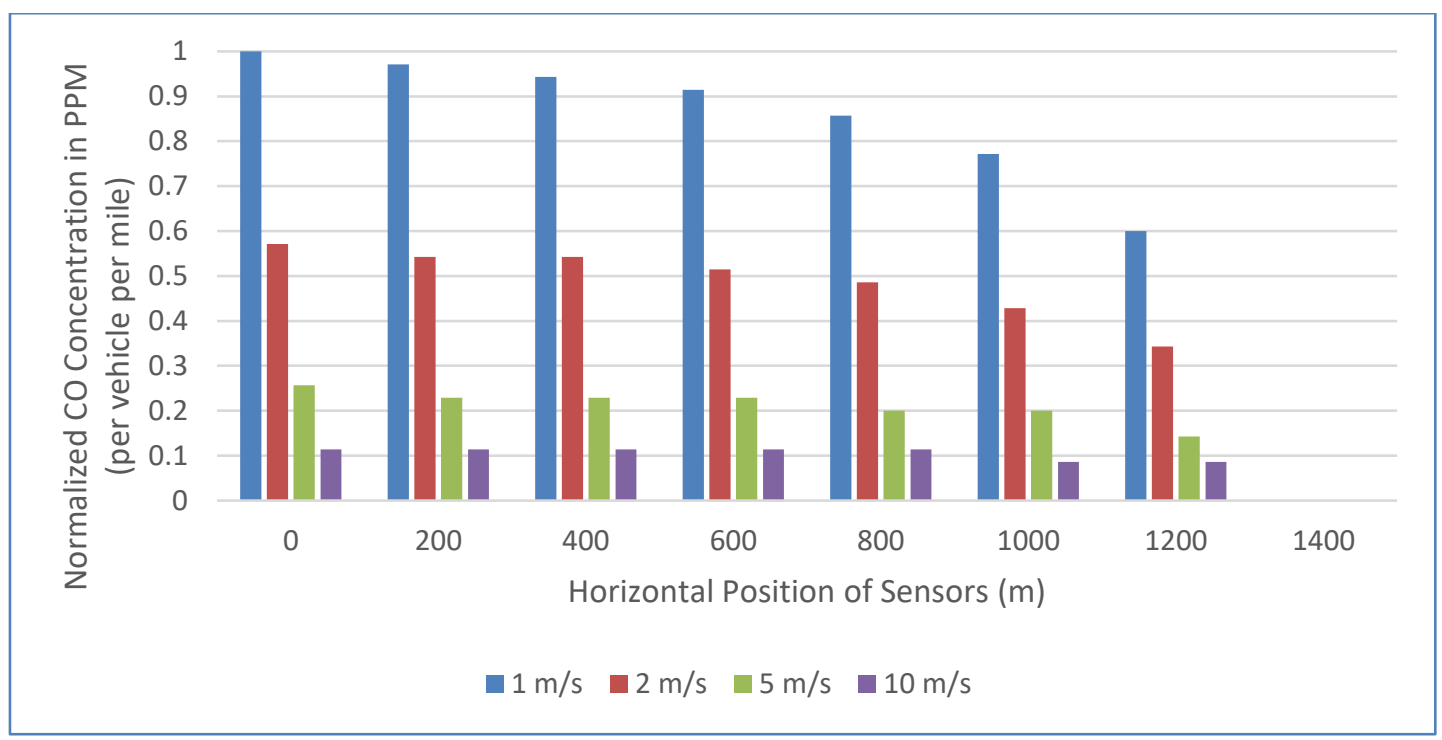

Fig. 2: Normalized CO Concentration at different wind speeds at minimum receptors' height (1.8 $\mathrm{m})$ and wind angle of 360 degrees (north wind)).

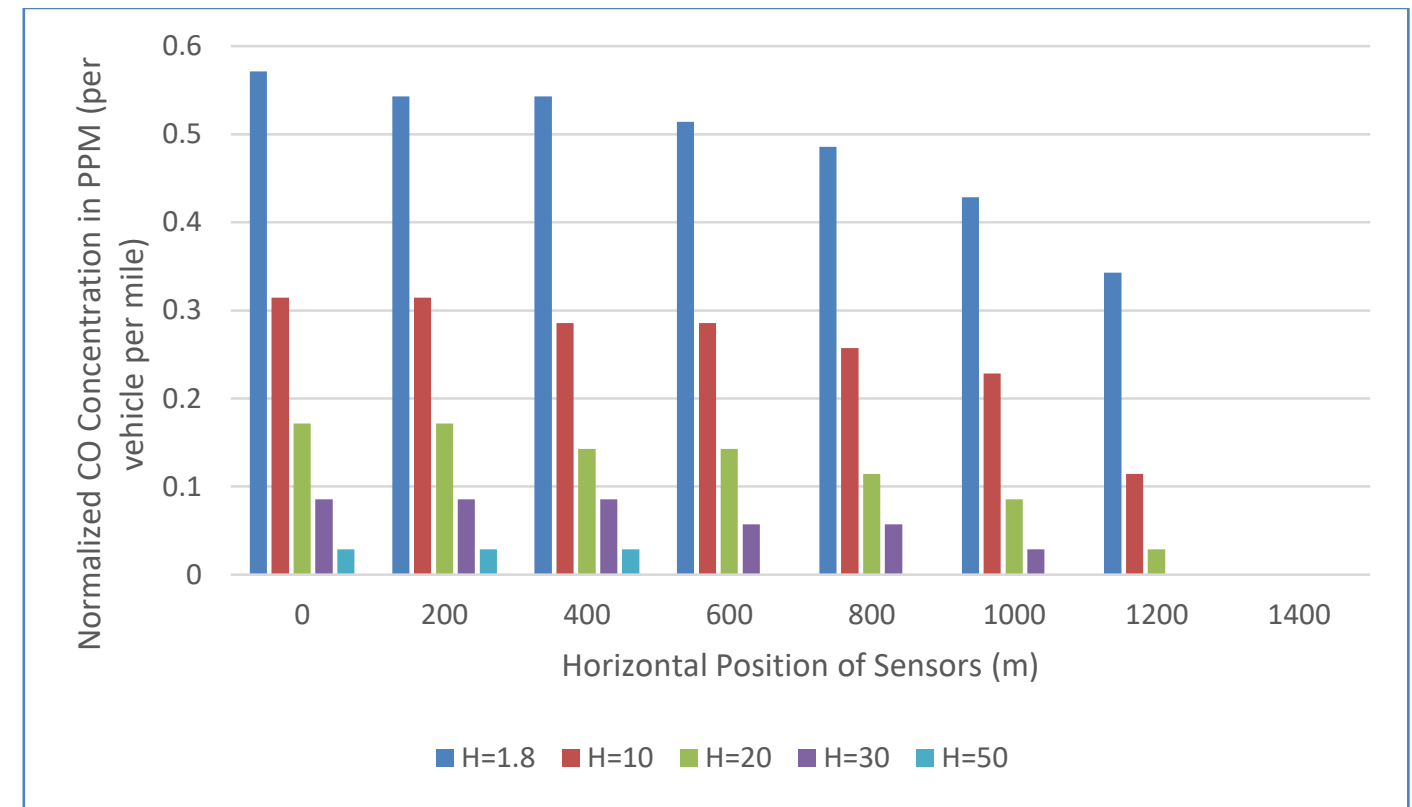

Fig. 3: Normalized CO Concentration at different heights $(\mathrm{H})$ with wind speed of $2 \mathrm{~m} / \mathrm{s}$ and wind angle 360 degrees (north wind), Monday at $4 \mathrm{PM}$. 


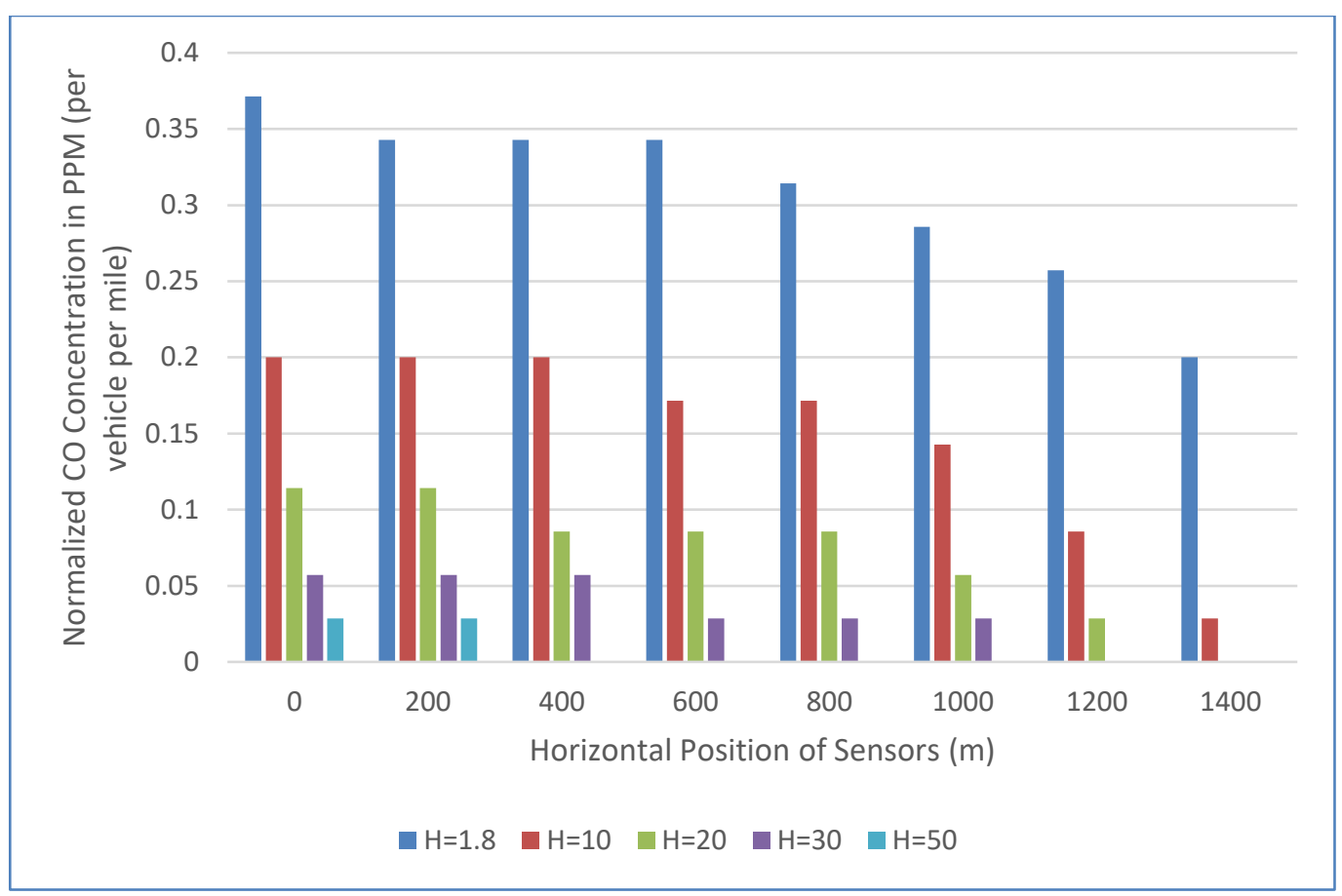

Fig. 4: Normalized CO Concentration at different heights $(\mathrm{H})$ with wind speed of $2 \mathrm{~m} / \mathrm{s}$ and wind angle of 360 degrees (north wind), Sunday at $4 \mathrm{PM}$.

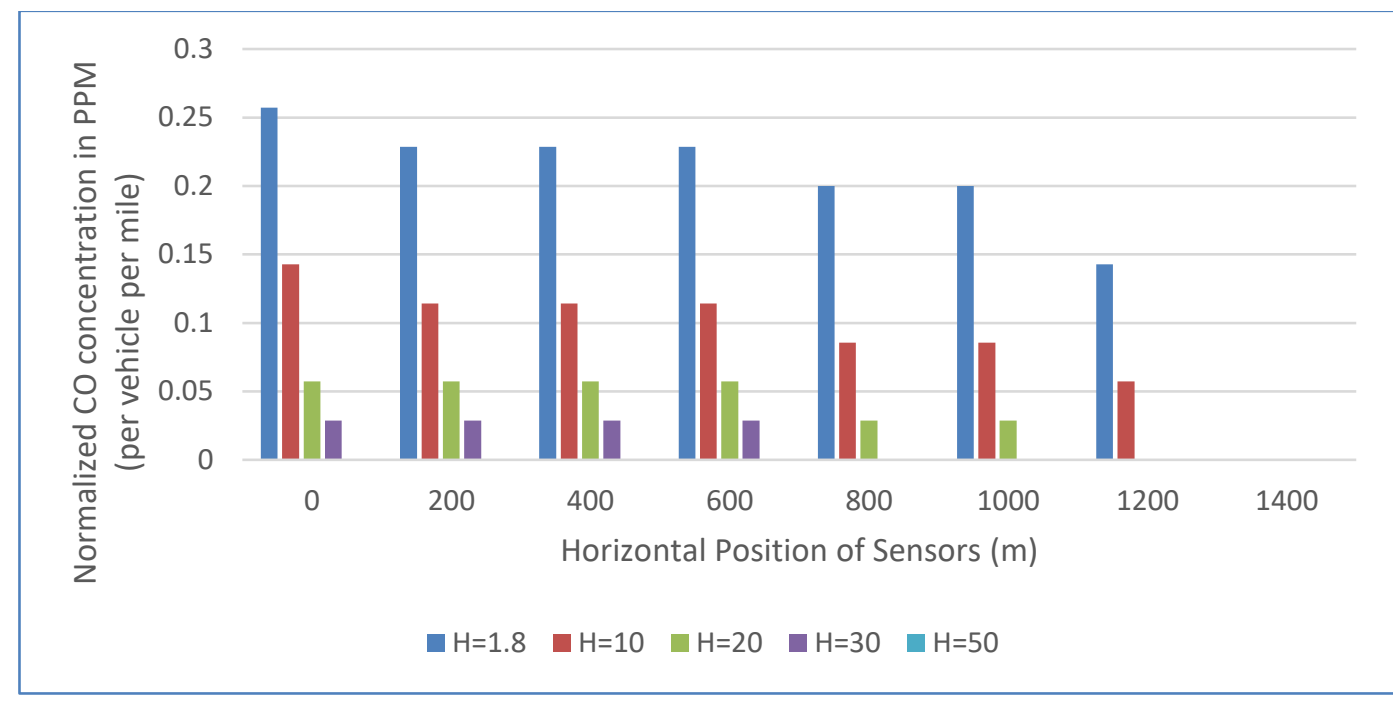

Fig. 5: Normalized CO Concentration at different heights $(\mathrm{H})$ with wind speed of $5 \mathrm{~m} / \mathrm{s}$ and wind angle 360 degrees (north wind), Monday 4 PM. 


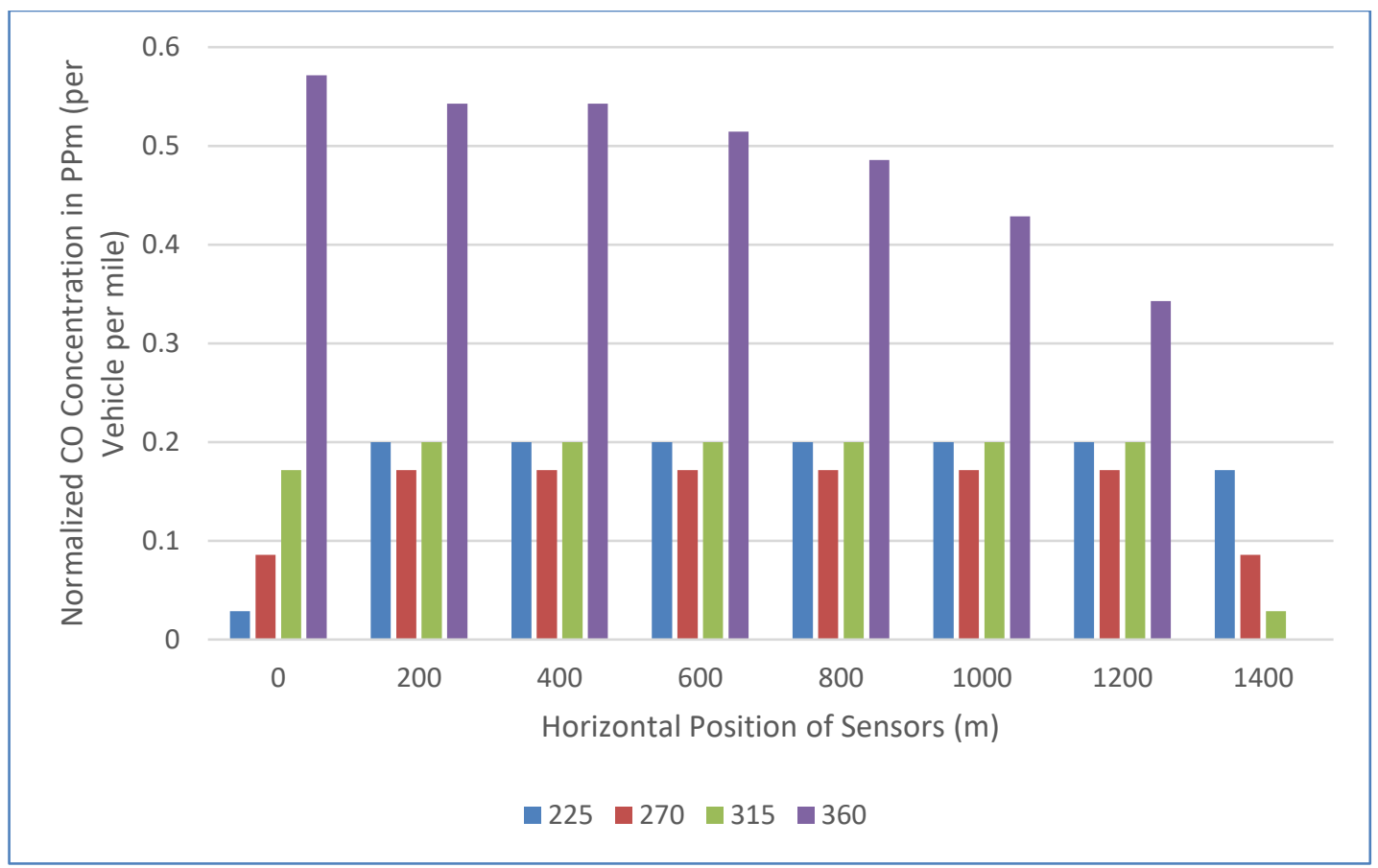

Fig. 6: Normalized CO Concentration with different wind angles at wind speed of $2 \mathrm{~m} / \mathrm{s}$ and minimum height (1.8 $\mathrm{m})$.

In Figure 2, we show the normalized CO concentration at different wind speeds with wind angle of 360 degrees and minimum receptor height of $1.8 \mathrm{~m}$. Since our results in [8] showed that $\mathrm{NO}_{\mathrm{x}}, \mathrm{HCs}$ and $\mathrm{CH}_{4}$ emission levels follow the same trend as $\mathrm{CO}$ emission levels but at lower values, we will focus in this study on estimating the $\mathrm{CO}$ concentration level at different receptors' locations. All the results, unless otherwise specified, are obtained using the MOVES CO emission levels obtained for Monday at 4 PM. All the values in Figure 2 and the subsequent figures are normalized to the maximum CO concentration which occurs at the lowest wind speed of $1 \mathrm{~m} / \mathrm{s}$ at minimum receptor height $(1.8 \mathrm{~m})$ and wind angle of 360 degrees. The wind speeds determine the amount of dispersion of pollutants in the atmosphere. The results in Figure 2 show that $\mathrm{CO}$ pollutant is more concentrated along the highway link at lower wind speeds.

In Figures 3 and 4, we show the normalized $\mathrm{CO}$ concentration at different receptor heights ( $\mathrm{Z}$ coordinate) with wind speed of $2 \mathrm{~m} / \mathrm{s}$ for Monday at $4 \mathrm{PM}$ and Sunday at 4PM, receptively. Both figures show that the CO concentration level depends on the vertical distance of the receptor above the ground and that lower pollutant concentration is obtained as the vertical distance of the receptors placed from the ground increases. Figure 4 has lower $\mathrm{CO}$ concentration levels as the emission levels obtained from MOVES per vehicle per mile were lower for Sunday at 4 PM compared to Monday at 4 PM [8]. Figure 5 shows the normalized $\mathrm{CO}$ concentration at different receptor heights ( $\mathrm{Z}$ coordinate) with wind speed of $5 \mathrm{~m} / \mathrm{s}$. This figure shows that lower pollutant concentration is obtained as the distance of the vertical receptor from the ground increases for different wind speeds.

Figure 6 shows the normalized CO concentration with different wind angles at wind speed of $2 \mathrm{~m} / \mathrm{s}$ and minimum height of $1.8 \mathrm{~m}$. The figure shows that the wind direction (angle) affects the highest pollutant concentration level obtained.

\section{Conclusions}

In this paper, we estimated the pollutant concentration at desired locations using the CAL3QHC air dispersion model coupled with emission levels obtained from MOVES. We examined the effect of wind speed, wind angle and sensors' heights on the pollutant concentration levels obtained. Simulation results showed that higher pollutant concentrations are obtained when the wind speed is low and the receptor height is near the emission source (receptor is placed closer to the ground). This information can be used to map out the sensor network locations (vertical and horizontal placement) for optimal sensor deployment to measure real time pollutant concentration levels. 


\section{Acknowledgements}

This research was supported by a grant from the Ontario Ministry of Transportation. Opinions expressed in this report are those of the authors and may not necessarily reflect the views and policies of the ministry. The authors would like to acknowledge MTO for sponsoring this project and providing the data used in the analysis and sharing their valuable experience and insights.

\section{References}

[1] R. M. Harrison, R. Tillinga, M. S. Romero, S. Harrad and K. Jarvis, "A study of trace metals and polycyclic aromatic hydrocarbons in the roadside environment," Atmospheric Environment, vol. 37, no. 17, pp. 2391-2402, 2003.

[2] J. J. Kim , S. Smorodinsky, M. Lipsett, B. C. Singer, A. T. Hodgson and B. Ostro "Traffic-related Air Pollution near Busy Roads: The East Bay Children's Respiratory Health Study," American Journal of Respiratory and Critical Care Medicine, vol. 170, no. 5, pp. 520-526, 2004.

[3] Canadian Council of Ministers of the Environment, "Canadian National Ambient Air Quality Objectives: Process and Status," Canadian Environmental Quality Guidelines, 1999.

[4] Canadian Council of Ministers of the Environment, "Guidance Document on Achievement Determination Canadian Ambient Air Quality Standards for Fine Particulate Matter and Ozone," 2012.

[5] U.S. Environmental Protection Agency. MOtor Vehicle Emission Simulator (MOVES). [Online] Available: https:/www.epa.gov/moves

[6] C. D. Cooper and F. C. Alley, Air Pollution Control, a Design Approach. Fourth Edition. Illinois: Waveland Press, 2002.

[7] P. E. Benson, "A review of the development and application of the CALINE3 and 4 models," Atmospheric Environment, vol. 26B, pp. 379-390, 1992.

[8] L. Khalid, S. Ali, V. Mashatan and B. Komisar, "Methodology for Monitoring Aerial Emissions from Highways," International Conference of Recent Trends in Environmental Science and Engineering, pp. 1-7, 2018.

[9] G. Amirjamshidi, T. Mostafa, A. Misra and M. Roorda "Integrated Model for Microsimulating Vehicle Emissions, Pollutant Dispersion and Population Exposure," Transportation Research Part D: Transport and Environment, vol. 18, pp. 16-24, 2013.

[10] G. Lee, S. G. Ritchie, R. Jayakrishnan, S. I. You, J-D. Saphores, O. A. Ogunseitan, "Assessing air quality and health benefits of the Clean Truck Program in the Alameda corridor, CA," Transportation Research Part A: Policy and Practice, vol. 46, no 8, pp. 1177-1193, 2012.

[11] S. Samaranayake, S. Glaser, D. Holstius, J. Monteil, K. Tracton, E. Seto, A. M. Bayen, "Real-time estimation of pollution emissions and dispersion from highway traffic," Computer-Aided Civil and Infrastructure Engineering, vol. 29, pp. 546-558, 2014.

[12] S.Yang, Y. Wu and J. Woolschlager, "Integrated Modeling Framework for Highway Traffic Pollution Estimation," American Journal of Environmental Sciences, vol. 12, no. 3, pp. 140-151, 2016. 